This item was submitted to Loughborough's Research Repository by the author.

Items in Figshare are protected by copyright, with all rights reserved, unless otherwise indicated.

Competitive analysis of interrelated price online inventory problems with demands

PLEASE CITE THE PUBLISHED VERSION

https://doi.org/10.1017/S144618111700013X

PUBLISHER

Cambridge University Press (CUP) @ Australian Mathematical Society

VERSION

AM (Accepted Manuscript)

LICENCE

CC BY-NC-ND 4.0

REPOSITORY RECORD

Han, Shuguang, Jueliang Hu, and Diwei Zhou. 2019. "Competitive Analysis of Interrelated Price Online Inventory Problems with Demands”. figshare. https://hdl.handle.net/2134/24398. 


\title{
Competitive Analysis of Interrelated Price Online Inventory Problems with Demands ${ }^{\star}$
}

\author{
Shuguang $\operatorname{Han}^{1} \star \star$, Jueliang $\mathrm{Hu}^{1}$, and Diwei Zhou ${ }^{2 \star \star \star}$ \\ 1.Department of Mathematics, Zhejiang Sci-Tech University,Hangzhou 310018, China \\ 2. Department of Mathematical Sciences, Loughborough University, Loughborough, \\ LE11 3TU, UK
}

\begin{abstract}
This paper investigates the interrelated price online inventory problems in which decisions as to when and how much to replenish must be made in an online fashion to meet some demand even without concrete knowledge of future prices. The objective of the decision maker is to minimize the total cost with the demands met. Two different types of demand are considered carefully, which are linearly related demand to price and exponentially related demand to price. In this paper, the prices are online with only the price range variation known in advance, which are interrelated with the preceding price. Two models of price correlations are investigated. Namely an exponential model and a logarithmic model. The corresponding algorithms of the problems are developed and the competitive ratio of the algorithms are also derived by the solutions of linear programming.
\end{abstract}

Keywords: interrelated prices; online inventory problem; competitive analysis

\section{Introduction}

In recent years, online problem and competitive algorithm theory have received increasing attention. With the Economic Order Quantity (EOQ) model proposed by Wilson [1] in 1934, the inventory theory has gradually developed. In the classical inventory problems, prices are generally assumed to be a constant or follow a probability distribution. Serel [2] studied the optimal ordering and pricing problem based on the interrelated demand and price in the rapid response system. Banerjee and Sharma [3] studied the inventory model with seasonal demand in two potentially replaceable market. Sana [4] put the EOQ model generalized to the case of perishable products with sensitive demand to price. Lin and Ho [5] studied the optimal ordering and pricing problem of the joint inventory model with sensitive demand to price based on the quantity discount. Kalymon [6] studies the problem with price dependency on previous prices where also demand

\footnotetext{
* This work was supported by the Natural Science Foundation of China (11201428, 11471286).

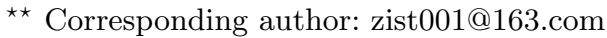

$\star \star \star$ Corresponding author: d.zhou2@lboro.ac.uk
} 
is uncertain. Webster and Weng [7] studied the ordering and pricing problem of the fashion product's supply chain which is consist of producer and seller, and random demand is sensitive to price in the supply chain. Ali and Masinga [8] presented a nonlinear programming model that can handle random demands and incorporate price change for optimal order quantities. Shu, Li and Zhong [9] considered an inventory control problem with stochastic demand in which the demand mean and variance are assumed known for each market. Liu, Song and $\mathrm{Wu}[10]$ investigated a single-period inventory problem with discrete stochastic demand. Yang, Wee, Chung and Huang [11] studied the product pricing and material replenishment strategy with price-sensitive demand. Sicilia et al. [12] studied an inventory model for deteriorating items with shortages and timevarying demand. Drezner and Scott [13] derived approximate formulas for the optimal solution for the particular case of an exponential demand distribution for the stochastic inventory model.

The price online inventory problem [14] is challenging where the decision maker, or a retailer, must decide when and how much to purchase without knowing future prices. The price online inventory problem can be seen as an extension of the time series search problem and the financial one-way trading problem [15-19], in which a decision maker wants to purchase $L$ units of product through a sequence of $n$ sellers $v_{1}, v_{2}, \ldots, v_{n}$ arriving online, and he needs to decide the fraction to purchase from each $v_{i}$ at the then-prevailing market price $p_{i}$. His objective is to minimize the cost. It is easy to solve the off-line version of the problem; if the decision maker knows all the future prices, he can simply wait for the lowest price and then purchase all his product at that price.

Specifically, in our price online inventory problem, there is a buyer who has $L$ units of product to be purchased, and there is a sequence of sellers $v_{1}, v_{2}, \ldots, v_{n}$ arriving. When a seller $v_{i}$ arrives, the unit price $p_{i}$ is revealed and the buyer needs to decide the amount $x_{i}$ of product to be purchased to $v_{i}$ at price $p_{i}$, and the objective is to minimize $\sum_{i} p_{i} x_{i}$ subject to $\sum_{i} x_{i} \leq L$. This optimisation problem is challenging because: (1) the buyer has no control of the prices, which fluctuates with time, and (2) the future prices are uninformative, i.e., when $v_{i}$ arrives, any price $p_{j}$ where $j>i$ is unknown, and (3) he needs to decide the amount of product to be purchased to a seller $v_{i}$ as soon as $v_{i}$ arrives. Larsen and Wohlk [14] considered a real-time version of the inventory problem with continuous deterministic demand and involved the fixed order cost, the inventory cost, obtained algorithmic upper and lower bounds of the competitive ratio whereas the gap grows with the complexity of the modes. The inventory problem considered in [20] is a demand online inventory problem where the decision maker only knows the upper bound and lower bound of the daily demand and decides how many products should be prepared everyday. Ma and Pan [21] considered the online inventory problem with the assumption that the decision maker has the knowledge of the same upper and lower bounds of all prices.

We apply the competitive ratio to evaluate the performance of algorithm. An arbitrary online algorithm $A L G$ is referred to as $r$-competitive, if for an arbitrary input price instance $I$ has $A L G(I) \leq r \cdot O P T(I)$, where $A L G(I)$ denotes 
the cost of the online algorithm $A L G$, and $O P T(I)$ is the cost of the optimal off-line algorithm $O P T$. The competitive ratio of $A L G$ algorithm is defined as the minimum $r$, which satisfies the above inequality.

To improve Larsen and Wohlk' work for a real-time inventory problem [14], we focus on two main facts of one inventory system, the price and the demand. The impacts of price, the price-related patterns and the relevant algorithms are discussed. In the Chinese stock market, the stock prices of today are known to be bounded in the interval from $90 \%$ to $110 \%$ of yesterday's closing price. So we modify the assumptions and assume that the variation range of each price is interrelated with its preceding price. In the inventory system there are some certain demands for the items, since there are some retailers and customers. The demand is negative correlated to the price because customers are more willing to purchase cheaper products. The problems considered in this paper become more practical than the problems in $[14,21]$. Two relationships and prices of demands considered, linear relation and exponential relation[23-25]. For every kind of demand, two types of price interrelation, exponential and logarithmic interrelations are considered, respectively[18-19].

\section{Problem Statement}

This paper considers an inventory problem in which the decision maker, a retailer, should decide when and how much to purchase every day without knowing future prices during the purchasing process. $U$ is the storage capacity, which must be reached when the purchasing process is over. Additionally, the initial inventory is zero. The objective of the decision maker is to minimize the total cost with the demands met. In order to generalise the model, we consider different price variation ranges. That is, the price has its own variation range and the range is variable.

Let $n$ denote the number of purchasing days. Denote by $D_{i}$ and $p_{i}$ the demand and the price of the $i$ th day. Let $\theta_{1}$ and $\theta_{2}$ denote the parameters of price variation ranges. We make some basic observation on the values of $\theta_{1}$ and $\theta_{2}$. If $1 \leq \theta_{1}$ or $\theta_{2} \leq 1$, implying that the price sequence is monotonously increasing or decreasing respectively, the optimal solution can be obtained by selecting the first or last price for the problems, respectively. So we just focus on the case where $0<\theta_{1} \leq 1 \leq \theta_{2}$. Let $p$ denote the initial price, where $p_{1} \in\left[\theta_{1} p, \theta_{2} p\right]$. As in the Chinese stock market, the stock prices follow the exponential model with $\theta_{1}=0.9$ and $\theta_{2}=1.1$. In the currency trading, the logarithmic-growth model was considered by Zhang, Zhang and $\mathrm{Xu}[19]$. The following two price interrelation models are considered.

- The exponential model[18]: $p_{i} \in\left[\theta_{1} p_{i-1}, \theta_{2} p_{i-1}\right], 2 \leq i \leq n$.

- The logarithmic model[19]: $p_{i} \in\left[\theta_{1} p_{1} \ln i, \theta_{2} p_{1} \ln i\right], 2 \leq i \leq n$. 


\section{The Competitive Analysis for Linearly Related Demand}

For the inventory problem, the demand is assumed to have a negative linear relation with price. Without loss of generality, we assume $D_{i}=a_{i}-b_{i} p_{i}$ as in the reference[23].

\subsection{The Competitive Analysis of the Exponential Model}

Now a linear programming problem with variables $\left\{r, s_{1}, s_{2}, \ldots, s_{n}\right\}$ as follows is investigated, where the second and third constraint conditions are transformed by the range of the total purchase quantity at the end of the $j_{t h}$ day $(j=$ $1,2, \ldots, n)$ and the relationship between the demand and price and the price correlation in the exponential model. The assumption of exponential model is $p_{i} \in\left[\theta_{1} p_{i-1}, \theta_{2} p_{i-1}\right]$ for every $2 \leq i \leq n$, and there exists one positive $p \in$ $\left[\theta_{1} p, \theta_{2} p\right]$ with $\theta_{1} \leq 1 \leq \theta_{2}$. The linear programming problem is given.

$$
\begin{array}{lll}
\min & r & \\
\text { s.t. } & H_{i}\left(s_{1}, s_{2}, \ldots, s_{n}\right) \leq r & \\
& U+\sum_{i=1}^{n}\left(a_{i}-b_{i} \theta_{2}^{i} p\right) \leq \sum_{i=1}^{n} s_{i} \leq U+\sum_{i=1}^{n}\left(a_{i}-b_{i} \theta_{1}^{i} p\right) & \\
& \sum_{i=1}^{j}\left(a_{i}-b_{i} \theta_{2}^{i} p\right) \leq \sum_{i=1}^{j} s_{i} \leq U+\sum_{i=1}^{j}\left(a_{i}-b_{i} \theta_{1}^{i} p\right) & j=1,2, \ldots, n-1 \\
& s_{i} \geq 0 & i=1,2, \ldots, n
\end{array}
$$

where $H_{i}\left(s_{1}, s_{2}, \ldots, s_{n}\right)=\frac{\frac{s_{1}}{\theta_{1}^{i-1}}+\frac{s_{2}}{\theta_{1}^{i-2}}+\ldots+\frac{s_{i-1}}{\theta_{1}}+s_{i}+s_{i+1} \theta_{2}+\ldots+s_{n} \theta_{2}^{n-i}}{U+\sum_{j=1}^{n}\left(a_{j}-b_{j} \theta_{2}^{j} p\right)}$.

Lemma 1. The solution to the linear programming problem $(I)$ exists.

Proof. It only needs to prove that there exists $\left\{r^{\prime}, s_{1}^{\prime}, s_{2}^{\prime}, \ldots, s_{n}^{\prime}\right\}$ such that

$$
\begin{gathered}
H_{i}\left(s_{1}^{\prime}, s_{2}^{\prime}, \ldots, s_{n}^{\prime}\right) \leq r^{\prime}, \quad i=1,2, \ldots, n \\
U+\sum_{i=1}^{n}\left(a_{i}-b_{i} \theta_{2}^{i} p\right) \leq \sum_{i=1}^{n} s_{i}^{\prime} \leq U+\sum_{i=1}^{n}\left(a_{i}-b_{i} \theta_{1}^{i} p\right), \\
\sum_{i=1}^{j}\left(a_{i}-b_{i} \theta_{2}^{i} p\right) \leq \sum_{i=1}^{j} s_{i}^{\prime} \leq U+\sum_{i=1}^{j}\left(a_{i}-b_{i} \theta_{1}^{i} p\right) \quad j=1,2, \ldots, n-1 \\
s_{i}^{\prime} \geq 0, \quad i=1,2, \ldots, n .
\end{gathered}
$$


We construct it as follows. Let $s_{1}^{\prime}=U+a_{1}-b_{1} \theta_{1} p$ and $s_{i}^{\prime}=a_{i}-b_{i} \theta_{1}^{i} p$ for every $2 \leq i \leq n$. It is obvious that $s_{i}^{\prime} \geq 0$ for $1 \leq i \leq n$ and $\sum_{i=1}^{n} s_{i}^{\prime}=U+\sum_{i=1}^{n}\left(a_{i}-b_{i} \theta_{1}^{i} p\right)$. And $\sum_{i=1}^{j} s_{i}^{\prime}=U+\sum_{i=1}^{j}\left(a_{i}-b_{i} \theta_{1}^{i} p\right)$ holds for $1 \leq j \leq n-1$. With the assumption $\theta_{1} \leq \theta_{2},\left\{s_{1}^{\prime}, s_{2}^{\prime}, \ldots, s_{n}^{\prime}\right\}=\left\{U+a_{1}-b_{1} \theta_{1} p, a_{2}-b_{2} \theta_{1}^{2} p, \ldots, a_{n}-b_{n} \theta_{1}^{n} p\right\}$ satisfies the inequalities (2), (3) and (4). In addition, for any $i=1,2, \ldots, n$, we get

$$
\begin{aligned}
& H_{i}\left(s_{1}^{\prime}, s_{2}^{\prime} \ldots, s_{n}^{\prime}\right) \\
& =\frac{\frac{U+a_{1}-b_{1} \theta_{1} p}{\theta_{1}^{i-1}}+\ldots+\frac{a_{i-1}-b_{i-1} \theta_{1}^{i-1} p}{\theta_{1}}+a_{i}-b_{i} \theta_{1}^{i} p+\left(a_{i+1}-b_{i+1} \theta_{1}^{i+1} p\right) \theta_{2}+\ldots+\left(a_{n}-b_{n} \theta_{1}^{n} p\right) \theta_{2}^{n-i}}{U+\sum_{j=1}^{n}\left(a_{j}-b_{j} \theta_{2}^{j} p\right)} .
\end{aligned}
$$

Because $\theta_{1}, \theta_{2}, U, n, p, a_{i}(1 \leq i \leq n)$ and $b_{i}(1 \leq i \leq n)$ are all known parameters, the values of $H_{i}\left(s_{1}^{\prime}, s_{2}^{\prime}, \ldots, s_{n}^{\prime}\right)=H_{i}\left(U+a_{1}-b_{1} \theta_{1} p, a_{2}-b_{2} \theta_{1}^{2} p, \ldots, a_{n}-b_{n} \theta_{1}^{n} p\right)$ can be calculated for all $1 \leq i \leq n$. Let $r^{\prime}=\max _{1 \leq i \leq n} H_{i}\left(U+a_{1}-b_{1} \theta_{1} p, a_{2}-\right.$ $\left.b_{2} \theta_{1}^{2} p, \ldots, a_{n}-b_{n} \theta_{1}^{n} p\right)$. It is clear that $H_{i}\left(U+a_{1}-b_{1} \theta_{1} p, a_{2}-b_{2} \theta_{1}^{2} p, \ldots, a_{n}-\right.$ $\left.b_{n} \theta_{1}^{n} p\right) \leq r^{\prime}$ for all $1 \leq i \leq n$. From the above analysis, there exists $\left\{r^{\prime}, s_{1}^{\prime}, s_{2}^{\prime}, \ldots, s_{n}^{\prime}\right\}=$ $\left\{\max _{1 \leq i \leq n} H_{i}\left(U+a_{1}-b_{1} \theta_{1} p, a_{2}-b_{2} \theta_{1}^{2} p, \ldots, a_{n}-b_{n} \theta_{1}^{n} p\right), U+a_{1}-b_{1} \theta_{1} p, a_{2}-b_{2} \theta_{1}^{2} p, \ldots, a_{n}-\right.$ $\left.b_{n} \theta_{1}^{n} p\right\}$ satisfying the inequalities (1), (2), (3) and (4). Thus, the solution of the above linear programming problem $(I)$ exists.

The online algorithm of this model is designed according to the solution to the linear programming problem $(I)$, denoted by $S L P_{1}$.

\section{Algorithm $\boldsymbol{S L P} \boldsymbol{P}_{1}$}

- Step 1: Solve the linear programming problem $(I)$, and let $\left\{r^{*}, s_{1}^{*}, s_{2}^{*}, \ldots, s_{n}^{*}\right\}$ be the solution.

- Step 2: Define $s_{i}^{*}$ to be the quantity of units for purchasing at period $i$ for every $1 \leq i \leq n$.

Theorem 1. The competitive ratio of $S L P_{1}$ algorithm is $r^{*}$.

Proof. Let $\sigma=p_{1}, p_{2}, \ldots, p_{n}$ be an arbitrary price sequence. Without loss of generality, we assume that the lowest price in $\sigma$ is $p_{i}$. Obviously, the optimal solution $O P T(\sigma) \geq\left(U+\sum_{j=1}^{n} D_{j}\right) p_{i}$ and $S L P_{1}(\sigma)=\sum_{j=1}^{n} s_{j}^{*} p_{j}$ hold.

For $p_{j} \in\left[\theta_{1} p_{j-1}, \theta_{2} p_{j-1}\right]$ when $2 \leq j \leq n$, then $p_{j} \leq \frac{p_{i}}{\theta_{1}^{i-j}}$ for $j=1,2, \ldots, i$, and $p_{j} \leq \theta_{2}^{j-i} p_{i}$ for $j=i+1, i+2, \ldots, n$. From $\frac{S L P_{1}(\sigma)}{O P T(\sigma)} \leq \frac{\sum_{j=1}^{n} s_{j}^{*} p_{j}}{\left(U+\sum_{j=1}^{n} D_{j}\right) p_{i}}$, one can 
get

$$
\begin{aligned}
\frac{S L P_{1}(\sigma)}{O P T(\sigma)} & \leq \frac{\frac{s_{1}^{*}}{\theta_{1}^{i-1}} p_{i}+\frac{s_{2}^{*}}{\theta_{1}^{i-2}} p_{i}+\ldots+\frac{s_{i-1}^{*}}{\theta_{1}} p_{i}+s_{i}^{*} p_{i}+s_{i+1}^{*} \theta_{2} p_{i}+\ldots+s_{n}^{*} \theta_{2}^{n-i} p_{i}}{\left(U+\sum_{j=1}^{n} D_{j}\right) p_{i}} \\
& \leq \frac{\frac{s_{1}^{*}}{\theta_{1}^{i-1}}+\frac{s_{2}^{*}}{\theta_{1}^{i-2}}+\ldots+\frac{s_{i-1}^{*}}{\theta_{1}}+s_{i}^{*}+s_{i+1}^{*} \theta_{2}+\ldots+s_{n}^{*} \theta_{2}^{n-i}}{U+\sum_{j=1}^{n}\left(a_{j}-b_{j} \theta_{2}^{j} p\right)} \\
& =H_{i}\left(s_{1}^{*}, s_{2}^{*}, \ldots, s_{n}^{*}\right)
\end{aligned}
$$

Combining the optimal solution to the linear programming problem $(I)$, the above inequality can be re-written in the following way.

$$
\frac{S L P_{1}(\sigma)}{O P T(\sigma)} \leq H_{i}\left(s_{1}^{*}, s_{2}^{*} \ldots, s_{n}^{*}\right) \leq r^{*}, \quad i=1,2, \ldots, n,
$$

where $r^{*}$ is the minimum one satisfying the above inequality. Hence, $r^{*}$ is the competitive ratio of the algorithm $S L P_{1}$.

\subsection{The Competitive Analysis of the Logarithmic Model}

The assumption of logarithmic model is $p_{i} \in\left[\theta_{1} p_{1} \ln i, \theta_{2} p_{1} \ln i\right]$ for $2 \leq i \leq n$, and there exists one positive $p$ satisfying $p_{1} \in\left[\theta_{1} p, \theta_{2} p\right]$ with $\theta_{1} \leq \theta_{2}$.

Let

$$
\begin{gathered}
K_{1}\left(s_{1}, s_{2}, \ldots, s_{n}\right)=\frac{s_{1}+s_{2} \theta_{2} \ln 2+\ldots+s_{n} \theta_{2} \ln n}{U+a_{1}-b_{1} \theta_{2} p+\sum_{j=2}^{n}\left(a_{j}-b_{j} \theta_{2}^{2} p \ln j\right)} \\
K_{i}\left(s_{1}, s_{2}, \ldots, s_{n}\right)=\frac{s_{1}+s_{2} \theta_{2} \ln 2+\ldots+s_{n} \theta_{2} \ln n}{\left(U+a_{1}-b_{1} \theta_{2} p+\sum_{j=2}^{n}\left(a_{j}-b_{j} \theta_{2}^{2} p \ln j\right)\right) \theta_{1} \ln i}, \quad i=2,3, \ldots, n .
\end{gathered}
$$

Before giving the competitive ratio, a linear programming problem with variables $\left\{r, s_{1}, s_{2}, \ldots, s_{n}\right\}$ is considered, in which the constraint conditions 2 to 4 are transformed by the range of the total purchase quantity at the end of the $j_{t h}$ 
day $(j=1,2, \ldots, n)$ in the logarithmic model.

$$
\begin{array}{llc}
\min & r & \\
\text { s.t. } & K_{i}\left(s_{1}, s_{2}, \ldots, s_{n}\right) \leq r & i=1,2, \ldots, n \\
& a_{1}-b_{1} \theta_{2} p \leq s_{1} \leq U+a_{1}-b_{1} \theta_{1} p & \\
& & \\
& a_{1}-b_{1} \theta_{2} p+\sum_{i=2}^{j}\left(a_{i}-b_{i} \theta_{2}^{2} p \ln i\right) \leq \sum_{i=1}^{j} s_{i} \leq U+a_{1}-b_{1} \theta_{1} p+\sum_{i=2}^{j}\left(a_{i}-b_{i} \theta_{1}^{2} p \ln i\right), \\
& & j=2, \ldots, n-1 \\
& U+a_{1}-b_{1} \theta_{2} p+\sum_{i=2}^{n}\left(a_{i}-b_{i} \theta_{2}^{2} p \ln i\right) \leq \sum_{i=1}^{n} s_{i} \leq U+a_{1}-b_{1} \theta_{1} p+\sum_{i=2}^{n}\left(a_{i}-b_{i} \theta_{1}^{2} p \ln i\right) \\
& s_{i} \geq 0 & i=1,2, \ldots, n
\end{array}
$$

Lemma 2. The solution to the linear programming problem $(I I)$ exists.

The online algorithm of this model is designed according to the solution to the linear programming problem $(I I)$, denoted by $S L P_{2}$.

Algorithm $\boldsymbol{S L P} \boldsymbol{P}_{2}$

- Step 1: Solve the linear programming problem $(I I)$, and let $\left\{\hat{r}, \hat{s_{1}}, \hat{s_{2}}, \ldots, \hat{s_{n}}\right\}$ be the solution.

- Step 2: Define $\hat{s}_{i}$ to be the quantity of units for purchasing at period $i$ for every $1 \leq i \leq n$.

Theorem 2. The competitive ratio of $S L P_{2}$ algorithm is $\hat{r}$.

Proof. Let $\sigma=p_{1}, p_{2}, \ldots, p_{n}$ denote an arbitrary price sequence. Without loss of generality, we assume that the lowest price in $\sigma$ is $p_{i}$. For $i=1, \operatorname{OPT}(\sigma) \geq$ $\left(U+a_{1}-b_{1} \theta_{2} p+\sum_{j=2}^{n}\left(a_{j}-b_{j} \theta_{2}^{2} p \ln j\right)\right) p_{1}$ and $S L P_{2}(\sigma)=\sum_{j=1}^{n} \hat{s_{j}} p_{j}$ hold. With the assumption of $p_{j} \in\left[\theta_{1} p_{1} \ln j, \theta_{2} p_{1} \ln j\right]$ for $2 \leq j \leq n, p_{j} \leq \theta_{2} p_{1} \ln j$ holds for every $j=2,3, \ldots, n$. Then

$$
\begin{aligned}
\frac{S L P_{2}(\sigma)}{O P T(\sigma)} & \leq \frac{\sum_{j=1}^{n} \hat{s_{j}} p_{j}}{\left(U+a_{1}-b_{1} \theta_{2} p+\sum_{j=2}^{n}\left(a_{j}-b_{j} \theta_{2}^{2} p \ln j\right)\right) p_{1}} \\
& \leq \frac{\hat{s_{1}} p_{1}+\hat{s_{2}} \theta_{2} \ln 2 p_{1}+\ldots+\hat{s_{n}} \theta_{2} \ln n p_{1}}{\left(U+a_{1}-b_{1} \theta_{2} p+\sum_{j=2}^{n}\left(a_{j}-b_{j} \theta_{2}^{2} p \ln j\right)\right) p_{1}} \\
& =\frac{\hat{s_{1}}+\hat{s_{2}} \theta_{2} \ln 2+\ldots+\hat{s_{n}} \theta_{2} \ln n}{U+a_{1}-b_{1} \theta_{2} p+\sum_{j=2}^{n}\left(a_{j}-b_{j} \theta_{2}^{2} p \ln j\right)} \\
& =K_{1}\left(\hat{s_{1}}, \hat{s_{2}}, \ldots, \hat{s_{n}}\right)
\end{aligned}
$$


For $2 \leq i \leq n$, we have $O P T(\sigma) \geq\left(U+a_{1}-b_{1} \theta_{2} p+\sum_{j=2}^{n}\left(a_{j}-b_{j} \theta_{2}^{2} p \ln j\right)\right) p_{i}$ and $S L P_{2}(\sigma)=\sum_{j=1}^{n} \hat{s_{j}} p_{j}$. By the assumptions of this model, $p_{i} \geq \theta_{1} p_{1} \ln i$ holds for $i=2,3, \ldots, n$ and $p_{j} \leq \theta_{2} p_{1} \ln j$ holds for $j=2,3, \ldots, n$. Then

$$
\begin{aligned}
\frac{S L P_{2}(\sigma)}{O P T(\sigma)} & \leq \frac{\sum_{j=1}^{n} \hat{s_{j}} p_{j}}{\left(U+a_{1}-b_{1} \theta_{2} p+\sum_{j=2}^{n}\left(a_{j}-b_{j} \theta_{2}^{2} p \ln j\right)\right) p_{i}} \\
& \leq \frac{\hat{s_{1}} p_{1}+\hat{s_{2}} \theta_{2} \ln 2 p_{1}+\ldots+\hat{s_{n}} \theta_{2} \ln n p_{1}}{\left(U+a_{1}-b_{1} \theta_{2} p+\sum_{j=2}^{n}\left(a_{j}-b_{j} \theta_{2}^{2} p \ln j\right)\right) \theta_{1} p_{1} \ln i} \\
& =\frac{\hat{s_{1}}+\hat{s_{2}} \theta_{2} \ln 2+\ldots+\hat{s_{n}} \theta_{2} \ln n}{\left(U+a_{1}-b_{1} \theta_{2} p+\sum_{j=2}^{n}\left(a_{j}-b_{j} \theta_{2}^{2} p \ln j\right)\right) \theta_{1} \ln i} \\
& =K_{i}\left(\hat{s_{1}}, \hat{s_{2}}, \ldots, \hat{s_{n}}\right)
\end{aligned}
$$

Combining the above two cases, we obtain

$$
\frac{S L P_{2}(\sigma)}{O P T(\sigma)} \leq K_{i}\left(\hat{s_{1}}, \hat{s_{2}}, \ldots, \hat{s_{n}}\right) \leq \hat{r}, \quad i=1,2, \ldots, n,
$$

where $\hat{r}$ is the minimum one satisfying the above inequality. Hence, $\hat{r}$ is the competitive ratio of the algorithm $S L P_{2}$.

\section{The Competitive Analysis for Exponentially Related Demand}

In this inventory problem, the demand is assumed to have a negative exponential relation with price. Without loss of generality, we assume $D_{i}=a_{i} \exp \left(-b_{i} p_{i}\right)$ from the references [23-25].

\subsection{The Competitive Analysis of the Exponential Model}

Firstly, a linear programming problem with variables $\left\{r, s_{1}, s_{2}, \ldots, s_{n}\right\}$ as following is investigated, in which the second and third constraint conditions are transformed by the range of the total purchase quantity at the end of the $j_{t h}$ 
day $(j=1,2, \ldots, n)$.

$\min r$

s.t. $\quad M_{i}\left(s_{1}, s_{2}, \ldots, s_{n}\right) \leq r \quad i=1,2, \ldots, n$

$U+\sum_{i=1}^{n} a_{i} \exp \left(-b_{i} \theta_{2}^{i} p\right) \leq \sum_{i=1}^{n} s_{i} \leq U+\sum_{i=1}^{n} a_{i} \exp \left(-b_{i} \theta_{1}^{i} p\right)$

$\sum_{i=1}^{j} a_{i} \exp \left(-b_{i} \theta_{2}^{i} p\right) \leq \sum_{i=1}^{j} s_{i} \leq U+\sum_{i=1}^{j} a_{i} \exp \left(-b_{i} \theta_{1}^{i} p\right) \quad j=1,2, \ldots, n-1$

$s_{i} \geq 0 \quad i=1,2, \ldots, n$

where $M_{i}\left(s_{1}, s_{2}, \ldots, s_{n}\right)=\frac{\frac{s_{1}}{\theta_{1}^{i-1}}+\frac{s_{2}}{\theta_{1}^{i-2}}+\ldots+\frac{s_{i-1}}{\theta_{1}}+s_{i}+s_{i+1} \theta_{2}+\ldots+s_{n} \theta_{2}^{n-i}}{U+\sum_{j=1}^{n} a_{j} \exp \left(-b_{j} \theta_{2}^{j} p\right)}$.

Lemma 3. The solution to the linear programming problem (III) exists.

The online algorithm of this model can be designed according to the solution to the linear programming problem $(I I I)$, and denoted by $S L P_{3}$.

Algorithm $\boldsymbol{S L P}_{3}$

- Step 1: Solve the linear programming problem $(I I I)$, and let $\left\{r^{*}, s_{1}^{*}, s_{2}^{*}, \ldots, s_{n}^{*}\right\}$ be the solution.

- Step 2: Define $s_{i}^{*}$ to be the quantity of units for purchasing at period $i$ for every $(1 \leq i \leq n)$.

Theorem 3. The competitive ratio of $S L P_{3}$ algorithm is $r^{*}$.

Proof. Let $\sigma=p_{1}, p_{2}, \ldots, p_{n}$ be an arbitrary price sequence. Without loss of generality, we assume that the lowest price in $\sigma$ is $p_{i}$. Obviously, $\operatorname{OPT}(\sigma) \geq$ $\left(U+\sum_{j=1}^{n} D_{j}\right) p_{i}$ and $S L P_{3}(\sigma)=\sum_{j=1}^{n} s_{j}^{*} p_{j}$ hold. For $2 \leq j \leq n, p_{j} \in\left[\theta_{1} p_{j-1}, \theta_{2} p_{j-1}\right]$ holds, then we have $p_{j} \leq \frac{p_{i}}{\theta_{1}^{i-j}}$ for $j=1,2, \ldots, i$ and $p_{j} \leq \theta_{2}^{j-i} p_{i}$ for $j=i+1, i+$ $2, \ldots, n$. From $\frac{S L P_{3}(\sigma)}{O P T(\sigma)} \leq \frac{\sum_{j=1}^{n} s_{j}^{*} p_{j}}{\left(U+\sum_{j=1}^{n} D_{j}\right) p_{i}}$, one can get

$$
\begin{aligned}
\frac{S L P_{3}(\sigma)}{O P T(\sigma)} & \leq \frac{\frac{s_{1}^{*}}{\theta_{1}^{i-1}} p_{i}+\frac{s_{2}^{*}}{\theta_{1}^{i-2}} p_{i}+\ldots+\frac{s_{i-1}^{*}}{\theta_{1}} p_{i}+s_{i}^{*} p_{i}+s_{i+1}^{*} \theta_{2} p_{i}+\ldots+s_{n}^{*} \theta_{2}^{n-i} p_{i}}{\left(U+\sum_{j=1}^{n} D_{j}\right) p_{i}} \\
& \leq \frac{\frac{s_{1}^{*}}{\theta_{1}^{i-1}}+\frac{s_{2}^{*}}{\theta_{1}^{i-2}}+\ldots+\frac{s_{i-1}^{*}}{\theta_{1}}+s_{i}^{*}+s_{i+1}^{*} \theta_{2}+\ldots+s_{n}^{*} \theta_{2}^{n-i}}{U+\sum_{j=1}^{n} a_{j} \exp \left(-b_{j} \theta_{2}^{j} p\right)} \\
& =M_{i}\left(s_{1}^{*}, s_{2}^{*}, \ldots, s_{n}^{*}\right)
\end{aligned}
$$


Combining the optimal solution to the linear programming problem (III), the above inequality can be rewritten in the following.

$$
\frac{S L P_{3}(\sigma)}{O P T(\sigma)} \leq M_{i}\left(s_{1}^{*}, s_{2}^{*}, \ldots, s_{n}^{*}\right) \leq r^{*}, \quad i=1,2, \ldots, n,
$$

where $r^{*}$ is the minimum one satisfying the above inequality. Hence, $r^{*}$ is the competitive ratio of the algorithm $S L P_{3}$.

\subsection{The Competitive Analysis of the Logarithmic Model}

Let

$$
\begin{gathered}
Q_{1}\left(s_{1}, s_{2}, \ldots, s_{n}\right)=\frac{s_{1}+s_{2} \theta_{2} \ln 2+\ldots+s_{n} \theta_{2} \ln n}{U+a_{1} \exp \left(-b_{1} \theta_{2} p\right)+\sum_{j=2}^{n} a_{j} \exp \left(-b_{j} \theta_{2}^{2} p \ln j\right)}, \quad, \quad(16) \\
Q_{i}\left(s_{1}, s_{2}, \ldots, s_{n}\right)=\frac{s_{1}+s_{2} \theta_{2} \ln 2+\ldots+s_{n} \theta_{2} \ln n}{\left(U+a_{1} \exp \left(-b_{1} \theta_{2} p\right)+\sum_{j=2}^{n} a_{j} \exp \left(-b_{j} \theta_{2}^{2} p \ln j\right)\right) \theta_{1} \ln i}, \quad i=2,3, \ldots, n .
\end{gathered}
$$

Before giving the competitive ratio, a linear programming problem with variables $\left\{r, s_{1}, s_{2}, \ldots, s_{n}\right\}$ is considered, in which the constraint conditions are transformed by the range of the total purchase quantity at the end of the $j_{t h}$ day $(j=1,2, \ldots, n)$.

$$
\begin{array}{clr}
\min & r & \\
\text { s.t. } & Q_{i}\left(s_{1}, s_{2}, \ldots, s_{n}\right) \leq r & i=1,2, \ldots, n \\
& a_{1} \exp \left(-b_{1} \theta_{2} p\right) \leq s_{1} \leq U+a_{1} \exp \left(-b_{1} \theta_{1} p\right) & j=2, \ldots, n-1 \\
& a_{1} \exp \left(-b_{1} \theta_{2} p\right)+\sum_{i=2}^{j} a_{i} \exp \left(-b_{i} \theta_{2}^{2} p \ln i\right) \leq \sum_{i=1}^{j} s_{i} & \\
& \sum_{i=1}^{j} s_{i} \leq U+a_{1} \exp \left(-b_{1} \theta_{1} p\right)+\sum_{i=2}^{j} a_{i} \exp \left(-b_{i} \theta_{1}^{2} p \ln i\right) & j=2, \ldots, n-1 \\
& U+a_{1} \exp \left(-b_{1} \theta_{2} p\right)+\sum_{i=2}^{n} a_{i} \exp \left(-b_{i} \theta_{2}^{2} p \ln i\right) \leq \sum_{i=1}^{n} s_{i} & \\
& \sum_{i=1}^{n} s_{i} \leq U+a_{1} \exp \left(-b_{1} \theta_{1} p\right)+\sum_{i=2}^{n} a_{i} \exp \left(-b_{i} \theta_{1}^{2} p \ln i\right) & \\
& s_{i} \geq 0 & i=1,2, \ldots, n
\end{array}
$$

Lemma 4. The solution to the linear programming problem $(I V)$ exists.

The online algorithm of this model can be designed according to the solution to the linear programming problem $(I V)$, and is denoted by $S L P_{4}$.

\section{Algorithm $\boldsymbol{S L P}_{4}$}


- Step 1: Solve the linear programming problem $(I V)$, and let $\left\{\bar{r}, \overline{s_{1}}, \overline{s_{2}}, \ldots, \overline{s_{n}}\right\}$ be the solution.

- Step 2: Define $\bar{s}_{i}$ to be the quantity of units for purchasing at period $i$ for every $1 \leq i \leq n$.

Theorem 4. The competitive ratio of $S L P_{4}$ algorithm is $\bar{r}$.

Proof. Let $\sigma$ denote an arbitrary price sequence. Without loss of generality, we assume that the lowest price in $\sigma$ is $p_{i}$. For $i=1$,

$$
O P T(\sigma) \geq\left(U+a_{1} \exp \left(-b_{1} \theta_{2} p\right)+\sum_{j=2}^{n} a_{j} \exp \left(-b_{j} \theta_{2}^{2} p \ln j\right)\right) p_{1}
$$

and $S L P_{4}(\sigma)=\sum_{j=1}^{n} \overline{s_{j}} p_{j}$ hold. For $2 \leq j \leq n, p_{j} \in\left[\theta_{1} p_{1} \ln j, \theta_{2} p_{1} \ln j\right]$, then $p_{j} \leq \theta_{2} p_{1} \ln j$ holds for every $j=2,3, \ldots, n$.

$$
\begin{aligned}
\frac{S L P_{4}(\sigma)}{O P T(\sigma)} & \leq \frac{\sum_{j=1}^{n} \overline{s_{j}} p_{j}}{\left(U+a_{1} \exp \left(-b_{1} \theta_{2} p\right)+\sum_{j=2}^{n} a_{j} \exp \left(-b_{j} \theta_{2}^{2} p \ln j\right)\right) p_{1}} \\
& \leq \frac{\overline{s_{1}} p_{1}+\overline{s_{2}} \theta_{2} \ln 2 p_{1}+\ldots+\overline{s_{n}} \theta_{2} \ln n p_{1}}{\left(U+a_{1} \exp \left(-b_{1} \theta_{2} p\right)+\sum_{j=2}^{n} a_{j} \exp \left(-b_{j} \theta_{2}^{2} p \ln j\right)\right) p_{1}} \\
& =\frac{\overline{s_{1}}+\overline{s_{2}} \theta_{2} \ln 2+\ldots+\overline{s_{n}} \theta_{2} \ln n}{U+a_{1} \exp \left(-b_{1} \theta_{2} p\right)+\sum_{j=2}^{n} a_{j} \exp \left(-b_{j} \theta_{2}^{2} p \ln j\right)} \\
& =Q_{1}\left(\overline{s_{1}}, \overline{s_{2}}, \ldots, \overline{s_{n}}\right)
\end{aligned}
$$

For $2 \leq i \leq n, \operatorname{OPT}(\sigma) \geq\left(U+a_{1} \exp \left(-b_{1} \theta_{2} p\right)+\sum_{j=2}^{n} a_{j} \exp \left(-b_{j} \theta_{2}^{2} p \ln j\right)\right) p_{i}$ and $S L P_{4}(\sigma)=\sum_{j=1}^{n} \overline{s_{j}} p_{j}$ hold. By the assumptions of this model, we get $p_{i} \geq$ 
$\theta_{1} p_{1} \ln i$ and $p_{j} \leq \theta_{2} p_{1} \ln j$ hold for $i=2,3, \ldots, n$ and $j=2,3, \ldots, n$. Then

$$
\begin{aligned}
\frac{S L P_{4}(\sigma)}{O P T(\sigma)} & \leq \frac{\sum_{j=1}^{n} \overline{s_{j}} p_{j}}{\left(U+a_{1} \exp \left(-b_{1} \theta_{2} p\right)+\sum_{j=2}^{n} a_{j} \exp \left(-b_{j} \theta_{2}^{2} p \ln j\right)\right) p_{i}} \\
& \leq \frac{\overline{s_{1}} p_{1}+\overline{s_{2}} \theta_{2} \ln 2 p_{1}+\ldots+\overline{s_{n}} \theta_{2} \ln n p_{1}}{\left(U+a_{1} \exp \left(-b_{1} \theta_{2} p\right)+\sum_{j=2}^{n} a_{j} \exp \left(-b_{j} \theta_{2}^{2} p \ln j\right)\right) \theta_{1} p_{1} \ln i} \\
& =\frac{\overline{s_{1}}+\overline{s_{2}} \theta_{2} \ln 2+\ldots+\overline{s_{n}} \theta_{2} \ln n}{\left(U+a_{1} \exp \left(-b_{1} \theta_{2} p\right)+\sum_{j=2}^{n} a_{j} \exp \left(-b_{j} \theta_{2}^{2} p \ln j\right)\right) \theta_{1} \ln i} \\
& =Q_{i}\left(\overline{s_{1}}, \overline{s_{2}}, \ldots, \overline{s_{n}}\right)
\end{aligned}
$$

Combining the above two cases, one can obtain

$$
\frac{S L P_{4}(\sigma)}{O P T(\sigma)} \leq Q_{i}\left(\overline{s_{1}}, \overline{s_{2}}, \ldots, \overline{s_{n}}\right) \leq \bar{r}, \quad i=1,2, \ldots, n,
$$

where $\bar{r}$ is the minimum one satisfying the above inequality. Hence, $\bar{r}$ is the competitive ratio of the algorithm $S L P_{4}$.

\section{Conclusions}

This paper investigates two models for the interrelated price online inventory problem with two kinds of demand. The corresponding algorithms are designed and the competitive ratios are derived for the exponential and the logarithmic model with the daily demand, respectively. In the future, it is interesting to consider the problem where both the price and demand are online. It is also challenging to investigate the online inventory problem where the price information is updated in real time.

\section{References}

1. R. H. Wilson. A scientific routine for stock control. Harvard Business Review, 13: 116-129, 1934.

2. D. A. Serel. Optimal ordering and pricing in a quick response system. International Journal of Production Economics, 121: 700-714, 2009.

3. S. Banerjee, A. Sharma. Inventory model for seasonal-demand with option to change the market. Computers \& Industrial Engineering, 59: 807-818, 2010.

4. S. S. Sana. Price-sensitive demand for perishable items-an EOQ model. Applied Mathematics and Computation, 217: 6248-6259, 2011.

5. Y. J. Lin, C. H. Ho. Integrated inventory model with quantity discount and pricesensitive demand. Top, 19: 177-188, 2011. 
6. B. A. Kalymon. Stochastic prices in a single-item inventory purchasing model. Operations Research, 19: 1434-1458, 1971.

7. S. Webster, Z. K. Weng. Ordering and pricing policies in a manufacturing and distribution supply chain for fashion products. International Journal of Production Economics, 114: 476-486, 2008.

8. M. M. Ali, L. Masinga. A nonlinear optimization model for optimal order quantities with stochastic demand rate and price change. Journal of Industrial and Management Optimization, 3: 139-154, 2007.

9. J. Shu, Z. Y. Li, W. J. Zhong. A market selection and inventory ordering problem under demand uncertainty. Journal of Industrial and Management Optimization, 7: 425-434, 2011.

10. W. Liu, S. J. Song, C. Wu. Single-period inventory model with discrete stochastic demand based on prospect theory. Journal of Industrial and Management Optimization, 8: 277-590, 2012.

11. Y. C. Yang, H. M. Wee, S. L. Chung, Y. Y. Huang. Pricing and replenishment strategy for a multi-market deteriorating product with time-varying andprice-sensitive demand. Journal of Industrial and Management Optimization, 9: 769-787, 2013.

12. J. Sicilia, M. Gonzalez-De-la-Rosa, J. Febles-Acosta, et al. An inventory model for deteriorating items with shortages and time-varying demand. International Journal of Production Economics, 155: 155-162, 2014.

13. Z. Drezner, C. Scott. Approximate and exact formulasfor the (Q,r) inventory model. Journal of Industrial and Management Optimization, 11: 135C144, 2015.

14. K. S. Larsen, S. Wohlk, Competitive analysis of the online inventory problem. European Journal of Operational Research, 207: 685-696, 2010.

15. R. El-Yaniv, et al. Optimal search and one-way trading online algorithms. Algorithmica, 30: 101-139, 2001.

16. Y. Xu, W. Zhang, F. Zheng, Optimal algorithms for the online time series search problem. Combinatorial Optimization and Applications, COCOA 2009, LNCS 5573: 322-333, 2009.

17. P. Damaschke, P. H. Ha, P. Tsigas, Online search with time-varying price bounds. European Journal of Operational Research, 55: 619-642, 2009.

18. W. Zhang, Y. Xu, F. Zheng, et al. Optimal algorithms for online time series search and one-way trading with interrelated prices. Journal of combinatorial optimization, 23: 159-166, 2012.

19. Y. Zhang, W. G. Zhang, W. J. Xu, Study on competitive strategies for online currency trading based on risk-aversion. Chinese Journal of Management, 7: 11021106, 2010.

20. W. M. Ma, G. Q. Chen, Competitive algorithms for the on-line inventory problem. Proceedings of the Third International Conference on Machine Learning and Cybernetics, 2: 898-903, 2004.

21. W. M. Ma, Y. Q. Pan, Competitive analysis of price uncertainty inventory problem. ICSESS, 179-182, 2011.

22. G. Schmidt, E. Mohr, M. Kersch, Experimental analysis of an online trading algorithm. Electronic Notes in Discrete Mathematics, 36: 519-526, 2010.

23. Z. Wang, S. Deng, Y. Ye. Close the gaps: a learning-while-doing algorithm for single-product revenue management problems. Operations Research, 62(2): 318-331, 2014.

24. Victor F. Araman, Ren Caldentey. Dynamic pricing for nonperishable products with demand learning. Operations Research, 57(5): 1169C1188, 2009.

25. Smith S.A.,Achabal D. Clearance pricing and inventory policies for retail chains. Management Science, 44: 285-300, 1998. 